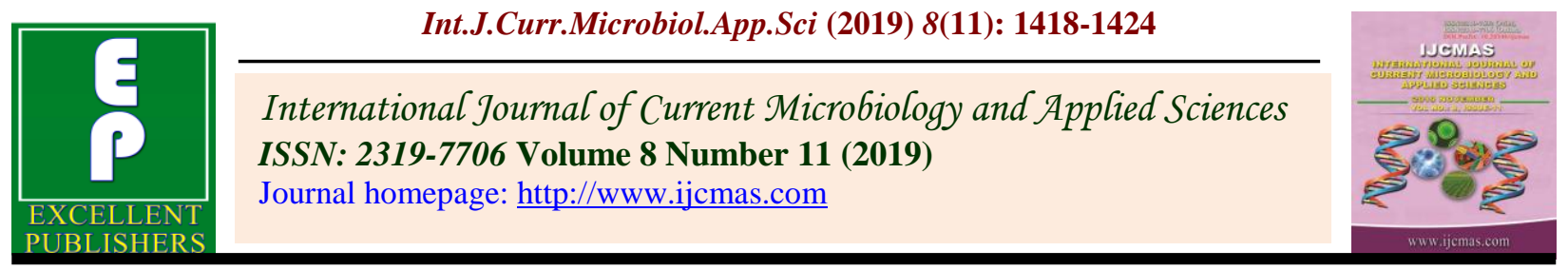

Original Research Article https://doi.org/10.20546/ijcmas.2019.811.166

\title{
Prevalence of Carbapenem Resistant Enterobacteriaceae in a Tertiary Care Hospital
}

\author{
Namitha Thomas and Tarana Sarwat*
}

Department of Microbiology, School of Medical Sciences and Research, Sharda University, Greater Noida, India

*Corresponding author

\section{A B S T R A C T}

Keywords

Enterobacteriaceae, Carbapenem resistance,

Escherichia coli, CRE prevalence

Article Info

Accepted:

12 October 2019

Available Online:

10 November 2019
Enterobacteriaceae which are a normal part of human gut bacteria are the common pathogens causing infections. Carbapenem resistant Enterobacteriaceae (CRE) is difficult to treat due to its high level of resistance to carbapenem. The present study was undertaken to determine the prevalence of carbapenem resistant Enterobacteriaceae in our hospital. Out of 519 clinical isolates identified with Enterobacteriaceae, 80 (18.54\%) were found to be carbapenem resistant. Imipenem showed complete resistance among CRE isolates. CRE was common in male patients $(53.75 \%)$ as well as urine samples $(52.1 \%)$. Escherichia coli were found as the predominant organism. This study indicates a high prevalence of CRE in our hospital emphasizing the need for implementing more effective infection control practices.

\section{Introduction}

Carbapenem resistance among Enterobacteriaceae is one of the foremost challenges that the medical world is facing since the past few years. ${ }^{[1]}$ Carbapenems were suggested as the final drug of choice for treatment during the emergence of ESBLs and AmpCs, due to their unique molecular structure and wide spectrum of activity. ${ }^{[2]}$ But the origin of carbapenemase enzyme in Enterobacteriaceae poses a major threat, creating a dilemma in therapeutic methods. ${ }^{[3]}$ These bacteria have the potential to cause wide spread transmission of resistance even between the intestinal bacteria ${ }^{[4]}$ through various intrinsic or extrinsic mechanisms. ${ }^{[5]}$

One major cause of resistance in bacteria is the production of carbapenem hydrolyzing enzyme with several variants, along with porin loss, production of AmpC and acquired MBL. ${ }^{[6]}$ Detection of these organisms is a difficult task due to the wide diversity in their 
resistance levels. ${ }^{[7]}$ The disc diffusion method is commonly used in the majority of laboratories. ${ }^{[8]}$ Commercially available chromogenic Media ${ }^{[2]}$ and various phenotypic and genotypic methods are also used for detection. ${ }^{[7]}$ Older but revived drugs alone or along with combination therapy are currently the treatment options, with several new alternatives. ${ }^{[9]}$

Studies conducted worldwide show an increasing pattern of Carbapenem resistance, especially in Enterobacteriaceae, Pseudomonas species and Acinetobacter species. ${ }^{[4]}$ Their prevalence varies according to regions with variation in genes. In the US and the Middle East infections by Acinetobacter baumannii and Pseudomonas aeruginosa are more prevalent, ${ }^{[10,11]}$ while in India, studies conducted over different period reports resistance in Enterobacteriaceae. ${ }^{[12]}$ To prevent and control the spread of outbreak improved infection control strategies have to be implemented in nosocomial settings. Early detection helps in proper treatment and isolation of patient preventing further spread as well as to prevent the wastage of antibiotics and reduce the financial burden on patients. [13]

\section{Materials and Methods}

The study was conducted in the Department of Microbiology, School of Medical Sciences \& Research, Sharda Hospital, Greater Noida from June 2018 to December 2018. The study population included all patients attending the outpatient departments and those admitted in various wards and ICUs of the Hospital. Samples like urine, stool, sputum, pus, blood, CSF, pleural fluid, peritoneal fluid, aspirates, etc. received for culture and sensitivity were included. The study was done with samples as per the predefined inclusion and exclusion criteria. The specimens were inoculated onto Blood agar and Mac Conkey agar and incubated overnight at $37^{\circ} \mathrm{C}$. Isolated colonies were subjected for identification by the characteristic appearance on respective media, Gram staining and were confirmed by the pattern of biochemical reactions using standard methods ${ }^{[123]}$. Ambiguous results were confirmed by the automated VITEK2 Compact system. Antimicrobial Susceptibility testing was performed for all the isolates (identified as/ belonging to Enterobacteriaceae family) on Muller-Hinton agar by KirbyBauer disc diffusion method as recommended by the Clinical Laboratory Standards Institute (CLSI) guidelines ${ }^{[13]}$. Organisms showing resistance one of the carbapenem drugs including imipenem, ertapenem, meropenem and doripenem with the susceptibility zones of $<=23 \mathrm{~mm}, 22 \mathrm{~mm}, 23 \mathrm{~mm}, 23 \mathrm{~mm}$ respectively were identified as carbapenem resistant.

\section{Results and Discussion}

Of the total samples received and processed, 519 isolates, identified as members of the Enterobacteriaceae family, were enrolled for the study. Out of the 519 samples, 147 were isolated from non-hospitalized patients and 372 from the hospitalized ones (Table 1). Eighty isolates out of the 519 isolates were confirmed as carbapenem resistant accounting for the prevalence of $18.54 \%$. Out of these 80 carbapenem resistant isolates 69 belonged to IPD patients and 11 to the OPD patients (Table 2). Gender-wise distribution of the patients with carbapenem resistant isolates, showed male predominance with male to female ratio 1.16:1 (Table 3). In categorization done based on age groups, 21-40 years $(36.25 \%)$ was predominant for CRE, followed by $41-65$ years $(30 \%), 0-2$ years $(11.25 \%)$ and $3-10$ years $(5 \%)$ (Table 4). Though overall male predominance was seen, variation existed between different age groups with female predominance in 21-40 years $(22.5 \%)$ and 41-65 years (17.5\%) (Table 5). Out of the CRE isolates Escherichia coli $(63.75 \%)$ was 
found to be highest followed by, Klebsiella pneumoniae $(11.25 \%)$, Klebsiella oxytoca (10\%), Citrobacter freundii (11.25\%), Citrobacter koseri $(2.5 \%)$ and Enterobacter species (1.25\%) (Table 6). Out of the four carbapenems tested not mentioned in methodology, the sensitivity rate was highest in Doripenem (48.75\%) followed by Meropenem (37.5\%), while the resistance was maximum with Imipenem (100\%) followed by Ertapenem (67.5\%) (Table 7). Among hospitalized patients, predominance of CRE in surgery unit $(36.23 \%)$ was maximum, followed by Medicine (27.53\%), Pediatrics (17.40\%), Obstetrics \& Gynecology (7.2\%) and others like respiratory medicine $(11.60 \%)$ (Table 8). From various samples tested, CRE was predominant in urine samples $(52.1 \%)$ followed by pus (20\%), stool (11.11\%) and sputum (10\%) and the least in other samples (6.25\%) like swabs, fluids, and tip from various sites of body (Table 9).

Infections with carbapenem resistant Enterobacteriaceae with limited treatment options are one of the major challenges that the health care setting is facing currently. Carbapenems are preferred over other antibiotics, due to their wide spectrum of activity and concentration-independent killing effect on bacteria, for treating invasive or lifethreatening conditions. However, over years organisms emerged with carbapenem resistance and continue to proliferate ${ }^{[1]}$. This study aimed at identifying the prevalence of CRE in our hospital.

Out of 519 samples identified with Enterobacteriaceae, $80 \quad(15.41 \%)$ were resistant to one or more carbapenems. Only limited data depicting the prevalence of carbapenem resistant Enterobacteriaceae was available from India, at the time of this study. This study depicts increased CRE prevalence (18.54\%) compared to that by Pravin K. Nair et al., ${ }^{[14]}$ with $12.26 \%$. Among various departments in the hospital, samples from the surgery unit (36.23\%) had the highest CRE rate compared to other units. It might be due to improper hygiene practices by the health care workers or patients, indicating the need for appropriate infection control practices within the hospital. CRE obtained from outpatient samples $(13.75 \%)$ suggests that it might be community-acquired, indicating significance in controlling the spread of CRE through faeco-oral route. Those with CRE can spread and contaminate the environment, especially in a country like India with about 1.4 million people as reservoirs. Therefore this finding alarms the need for investigation at the community level to identify and take appropriate measures ${ }^{[15]}$.

In our study, male $(53.75 \%)$ predominance was observed, resembling reports by T.V. Parimala $(50.90 \%)^{[16]}$ and Satyajeet K. Pawar et al., $(65.3 \%)^{[17]}$. Amongst different age groups included in the study, 21-40 years (36.25\%) had the most number of CRE followed by 41-65 years (30\%).

Contradictory to the male predominance as per our study, the female ratio was dominant in these age groups. In a study conducted by Kastor et al., non-communicable diseases are said to be the major cause of death and hospitalization in those aged above 35 years [18].

This factor points out that the cause of infection can be nosocomial as visible in this study. From overall samples included, CRE isolates were predominantly obtained from urine $(52.1 \%)$, identical to the study conducted by Nair et al., (46\%) and T.V Parimala $(55.26 \%)$. This elevation might be due to the proximity; Enterobacteriaceae being the major member of gut flora can be transmitted through faeco-oral route. Another possible reason is the prevalence of urinary tract infection in hospitalized patients ${ }^{[19]}$. 
Table.1 Distribution in hospitalized and non-hospitalized patients

\begin{tabular}{|c|c|c|}
\hline IPD/OPD & NUMBER & PERCENTAGE \\
\hline IPD & 372 & $86.25 \%$ \\
\hline OPD & 147 & $13.75 \%$ \\
\hline
\end{tabular}

Table.2 Distribution based on $C R E$ isolation from hospitalized and non-hospitalized patients

\begin{tabular}{|c|c|c|c|}
\hline IPD/OPD & TOTAL & POSITIVE & PERCENTAGE \\
\hline IPD & 372 & 69 & $18.54 \%$ \\
\hline OPD & 147 & 11 & $7.49 \%$ \\
\hline
\end{tabular}

Table.3 Showing gender-wise distribution of CRE

\begin{tabular}{|c|c|c|}
\hline GENDER & NUMBER & PERCENTAGE \\
\hline FEMALE & 37 & $46.25 \%$ \\
\hline MALE & 43 & $53.75 \%$ \\
\hline
\end{tabular}

Table.4 Showing age-wise distribution of CRE

\begin{tabular}{|c|c|c|}
\hline AGE GROUP & NUMBER & PERCENTAGE \\
\hline 0-2 years & 9 & $11.25 \%$ \\
\hline 3-10 years & 4 & $5 \%$ \\
\hline 11-20 years & 6 & $7.5 \%$ \\
\hline 21-40 years & 29 & $36.25 \%$ \\
\hline 41-65 years & 24 & $30 \%$ \\
\hline 65 years \& above & 8 & $10 \%$ \\
\hline
\end{tabular}

Table.5 Showing comparison of gender and age among CRE patients

\begin{tabular}{|c|c|c|c|c|}
\hline AGE GROUP & MALE & FEMALE & MALE \% & FEMALE \% \\
\hline 0-2 years & 8 & 1 & $10 \%$ & $1.25 \%$ \\
\hline 3-10 years & 2 & 2 & $2.5 \%$ & $2.5 \%$ \\
\hline $\mathbf{1 1 - 2 0}$ years & 4 & 2 & $5 \%$ & $2.5 \%$ \\
\hline $\mathbf{2 1 - 4 0}$ years & 11 & 18 & $13.75 \%$ & $22.5 \%$ \\
\hline $\mathbf{4 1 - 6 5}$ years & 10 & 14 & $12.5 \%$ & $17.5 \%$ \\
\hline 65 years \& above & 8 & 0 & $10 \%$ & $0 \%$ \\
\hline
\end{tabular}

Table.6 showing distribution of isolated microorganisms

\begin{tabular}{|c|c|c|}
\hline ORGANISM & NUMBER & PERCENTAGE \\
\hline Klebsiella oxytoca & 8 & $10 \%$ \\
\hline Klebsiella pneumoniae & 9 & $11.25 \%$ \\
\hline Escherichia coli & 51 & $63.75 \%$ \\
\hline Citrobacter freundii & 9 & $11.25 \%$ \\
\hline Citrobacter koseri & 2 & $2.5 \%$ \\
\hline Enterobacter species & 1 & $1.25 \%$ \\
\hline
\end{tabular}


Table.7 Showing the susceptibility pattern amongst carbapenems

\begin{tabular}{|c|c|c|c|c|}
\hline SUSCEPTIBILITY & ERTAPENEM & IMIPENEM & MEROPENEM & DORIPENEM \\
\hline Sensitive & 14 & 0 & 30 & 39 \\
\hline Resistant & 54 & 80 & 41 & 34 \\
\hline
\end{tabular}

Table.8 Showing CRE distribution in various departments of hospital

\begin{tabular}{|c|c|c|}
\hline DEPARTMENTS & NUMBER & PERCENTAGE \\
\hline Medical Unit & 19 & $27.53 \%$ \\
\hline Paediatric Unit & 12 & $17.40 \%$ \\
\hline Surgery Unit & 25 & $36.23 \%$ \\
\hline Obstetrics \& Gynaecology Unit & 5 & $7.24 \%$ \\
\hline Others & 8 & $11.60 \%$ \\
\hline
\end{tabular}

Table.9 Showing distribution of CRE in various samples

\begin{tabular}{|c|c|c|}
\hline SAMPLE & NUMBER & PERCENTAGE \\
\hline Urine & 42 & $52.1 \%$ \\
\hline Stool & 9 & $11.11 \%$ \\
\hline Sputum & 8 & $10 \%$ \\
\hline Pus & 16 & $20 \%$ \\
\hline Others & 5 & $6.25 \%$ \\
\hline
\end{tabular}

In our study, Escherichia coli was the predominant organism, followed by Klebsiella species, which is concordant with the study by Parimala and Prasanth Manohar et al., with $63.04 \%$ and $48 \%$ respectively ${ }^{[20]}$. In contrast, the study conducted by Wattal et al., (31\% wards and 51\% ICU) ${ }^{[21]}$ and Satyajeet K Pawar (63\%), displays predominance by Klebsiella species, showing regional variation in prevalence. The predominance by Escherichia coli might be due to the increased urine samples and Escherichia coli being a major urinary pathogen ${ }^{[22]}$.

Of the carbapenems tested, imipenem has a $100 \%$ resistance rate and doripenem is most sensitive $(48.75 \%)$. Some studies suggest imipenem has better activity than ertapenem [23], contrary to ours. Since treatment options for CRE are limited either monotherapy or combination therapy is considered ${ }^{[24]}$. In our study, polymyxins tested showed a 100\% sensitivity to CRE isolates.

One main limitation of our study was the inability to confirm CRE using any of the standard methods. Therefore the actual mechanism of resistance couldn't be detected and lack of molecular study caused unidentification of the gene prevalence. A study based on combination therapy was not performed, limiting the knowledge if monotherapy or combination therapy could be a better option for treating CRE.

The remedy for this menace of carbapenem resistance can be obtained by strict adherence to infection control strategies and policy guidelines designed for individual countries. From basic steps like hand hygiene, the performance of efficient identification tests in 
the clinical microbiology laboratory play an important role in the control of CRE infections. Earlier the detection of carbapenemase-producing isolates, from clinical infections and the carrier-states, lesser will be the development of untreatable infections. Also, knowledge about the prevalence and resistance pattern of an organism at a particular demographic zone will help in preparing local infection control guidelines.

\section{References}

Codjoe FS, Donkor ES. (2018). Carbapenem Resistance: A Review. Medical Sciences. 6(1):1.

Dahab RA et al., (2014) Detection of Carbapenem Resistant Gram-Negative Bacilli from Infected Wounds in Khartoum State-2014. Clin Microbiol 6:296.

Teo JQM, Cai Y, Lim T-P, Tan TT, Kwa ALH. (2016). Carbapenem Resistance in Gram-Negative Bacteria: The Not-SoLittle Problem in the Little Red Dot. Poirel L, ed. Microorganisms. 4(1):13.

Guidelines for the prevention and control of carbapenem-resistant Enterobacteriaceae, Acinetobacter baumannii and Pseudomonas aeruginosa in health care facilities. Geneva: World Health Organization; 2017. Licence: CC BY-NC-SA 3.0 IGO.

Raghunath, D. J. (2008). Emerging antibiotic resistance in bacteria with special reference to India. Biosci 33; 4; 593-603.

Pfeifer, Y, Cullik, A, Witte, W. (2010). Resistance to cephalosporins and carbapenems in gram-negative bacterial pathogens. Int $\mathrm{J}$ Med MicroBiol. 300:371-379.

Gazin M, Paasch F, Goossens H, MalhotraKumar S. (2012). Current Trends in
Culture-Based and Molecular Detection of Extended-Spectrum- $\beta$ Lactamase-Harboring and Carbapenem Resistant Enterobacteriaceae. Journal of Clinical Microbiology. 50(4):11401146.

CLSI. Performance Standards for Antimicrobial Susceptibility Testing. $28^{\text {th }}$ ed. CLSI supplement M100. Wayne, PA: Clinical and Laboratory Standards Institute; 2018.

Taneja N, Kaur H. (2016). Insights into Newer Antimicrobial Agents Against Gramnegative Bacteria. Microbiology Insights. 9: 9-19.

Cai B, Echols R, Magee G, et al., (2017). Prevalence of Carbapenem-Resistant Gram-Negative Infections in the United States Predominated by Acinetobacter baumannii and Pseudomonas aeruginosa. Open Forum Infectious Diseases. 4(3):176.

Porwal R, et al., (2014). Carbapenem resistant Gram-negative bacteremia in an Indian intensive care unit: A review of the clinical profile and treatment outcome of 50 patients. Indian Journal of Critical Care Medicine: Peer-reviewed, Official Publication of Indian Society of Critical Care Medicine. 18(11):750753.

Sekar R, Srivani S, Amudhan M, Mythreyee M. (2016). Carbapenem resistance in a rural part of southern India: Escherichia coli versus Klebsiella spp. The Indian Journal of Medical Research. 144(5):781-783.

Kaur M, Gupte S, Kaur T (2014) Detection of Carbapenem Resistant Gram-Negative Bacteria in Clinical Isolates from a Tertiary Care Hospital. J Bacteriol Mycol Open Access 2(1): 00011.

Nair P. K, et al., (2013). Carbapenem resistant Enterobacteriaceae from a tertiary hospital. J Microbiol Infect Dis 3 (4): 207-210. 
Nordmann P, Naas T, Poirel L. (2011).Global Spread of Carbapenemase-producing Enterobacteriaceae. Emerg Infect Dis 17:1791-1798.

T.V. Parimala. (2017). Screening of Carbapenem Resistant Enterobacteriaceae among Nosocomial Isolates: A Study from South India. Int.J.Curr.Microbiol.App.Sci 6(4): 460-465.

Satyajeet K Pawar et al., (2018) Carbapenem resistant Enterobacteriaceae: Prevalence and bacteriological profile in a tertiary teaching hospital from rural western India. Indian J Microbiol Res. 5(3): 342-347.

Kastor, Anshul and Sanjay K Mohanty. (2018). "Disease and age pattern of hospitalisation and associated costs in India: 1995-2014" BMJ open vol. 8, 24 Jan. doi:10.1136/bmjopen-2017016990.

Najar, M S et al., (2009). "Approach to urinary tract infections" Indian journal of nephrology vol. 19, 4: 129-39.
Manohar et al., (2017). The distribution of carbapenem- and colistin-resistance in Gram-negative bacteria from the Tamil Nadu region in India. Journal of Medical Microbiology 66:874-883.

Wattal C, et al., (2010). Surveillance of multidrug resistant organisms in tertiary care hospital in Delhi, India. J Assoc Physicians India. 58:32-36.

Stamm WE. (2001).An Epidemic of Urinary Tract Infections? N Engl J Med. 345:1055-1057.

Zhanel, G.G., Wiebe, R., Dilay, L. et al., (2007).Comparative review of Carbapenems. Drugs 67: 1027.

Matthew E. Falagas et al., (2014). Antibiotic Treatment of Infections Due to Carbapenem-Resistant Enterobacteriaceae: Systematic Evaluation of Available Evidence. Antimicrobial Agents and Chemotherapy 58 (2) 654-663.

\section{How to cite this article:}

Namitha Thomas and Tarana Sarwat. 2019. Prevalence of Carbapenem Resistant Enterobacteriaceae in A Tertiary Care Hospital. Int.J.Curr.Microbiol.App.Sci. 8(11): 14181424. doi: https://doi.org/10.20546/ijcmas.2019.811.166 